\title{
Acalculous adenomyomatosis of the gallbladder $^{1}$
}

\author{
G. BEVAN ${ }^{2}$ \\ From the Department of Medicine, UCLA School of Medicine, Center for the Health Sciences, \\ Los Angeles, California, USA
}

SUMMARY The course of acalculous adenomyomatosis of the gallbladder in six patients is. described. It is suggested that, even in the absence of gallstones, cholecystectomy should be advised when this condition is demonstrated radiographically in symptomatic subjects. The cause of the pain is unknown but it is probably related to excessive neuromuscular activity of the hyperplastic gallbladder wall.

One of the patients was found also to have an adenomatous polyp containing areas of adenocarcinoma. Although this polyp was not situated within an area of adenomyomatosis, it is possible that, as in most other patients with carcinoma of the gallbladder, previous disease may have predisposed to malignant change.

Adenomyomatosis is an uncommon abnormality of the gallbladder characterized by hyperplasia of the muscle layer and of the mucosa. Branched tubular projections of the mucosa into the muscle layer, Rokitansky-Aschoff sinuses, produce a histological appearance simulating gland and cyst formation thus giving rise to the earlier name of cholecystitis glandularis proliferans cystica (King and MacCallum, 1931). Aspects of the anatomical, pathological, and radiological features of adenomyomatosis have been reviewed on several occasions (King and MacCallum, 1931 ; Le Quesne and Ranger, 1957; Jutras, Longtin, and Lévesque, 1960; Jutras and Lévesque, 1966).

The clinical significance and management of this condition continue to provoke controversy. Because of the frequency with which some degree of adenomyomatosis may be found in prospective necropsy surveys, as high as $7 \%$ in one report (Bricker and Halpert, 1963), it is clear that in most instances the lesion does not produce symptoms. The diagnosis is frequently made by the radiologist on the basis of finding a small dimpled filling defect at the fundus of the gallbladder, often

Received for publication 17 June 1970.

${ }^{1}$ This work was supported in part by USPHS grant no. GRSG 05354.

'Present address: Edgware General Hospital, Edgware, Middlesex. accompanied by a kinking deformity of the body, or by a more generalized irregularity of the outline of the gallbladder caused by contrast material entering the Rokitansky-Aschoff sinuses. Gallstones commonly coexist with these changes so that biliary tract symptoms when they occur are usually ascribed to cholelithiasis with or without associated inflammation. In the absence of gallstones, however, many surgeons find it difficult to accept that symptoms may arise from simple adenomyomatosis, especially if gallbladder function appears normal radiologically, as it frequently does.

It is the purpose of the present report to emphasize that acalculous adenomyomatosis can be associated with symptoms which may be chred by surgery. In addition, the unsuspected presence of a carcinoma in one of these patients provides reason for caution in assuming that this condition always follows a benign course.

\section{Case 1}

M.E., a 36-year-old woman, had experienced episodes of postprandial right upper quadrant pain for 11 years. The pain was typical of 


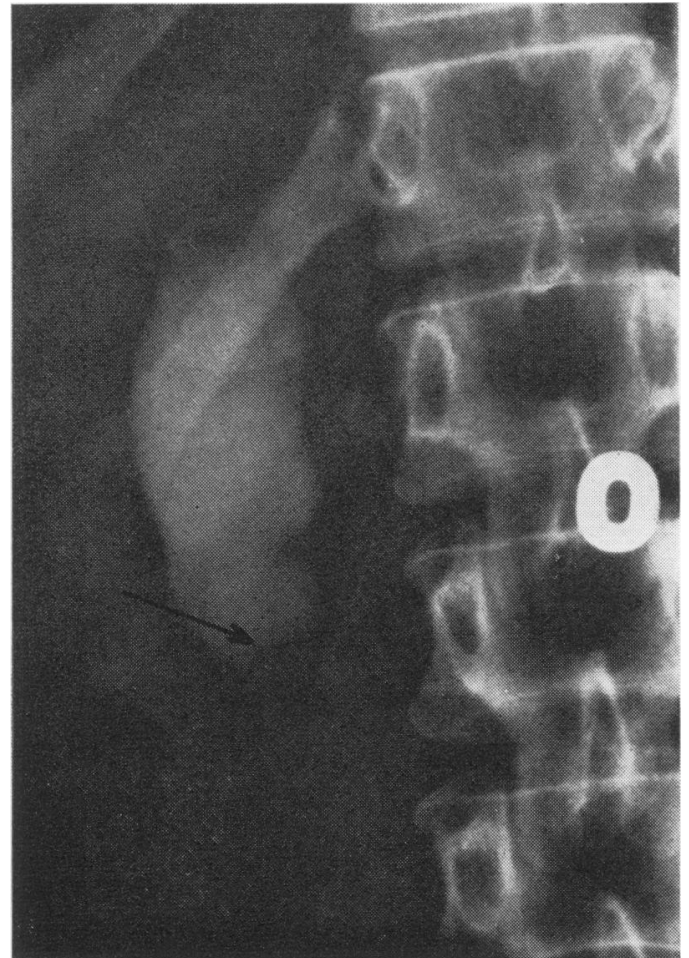

Fig. 1 Cholecystogram from patient 1 performed in 1968. A filling defect is visible in the fundus (see also Fig. 2).

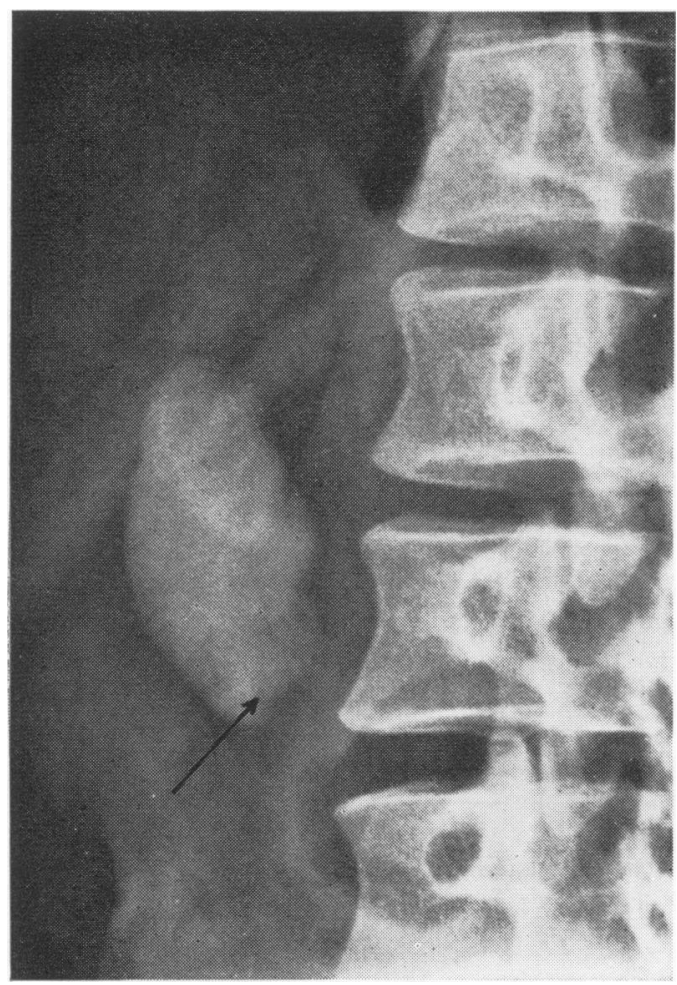

Fig. 2 Cholecystogram from patient 1 performed in 1961. biliary colic. It reached its maximum intensity soon after onset, it was constant in character, $\Omega$ radiated into the back between the shoulder $\rightleftharpoons$ blades, and was frequently accompanied by nausea $\overrightarrow{\vec{S}}$ and vomiting. There was no history of jaundice, $\frac{\vec{T}}{0}$ fever, or change in colour of stools or urine. She $\frac{}{0}$ had undergone radiological examination of the $\frac{\bar{\sigma}}{\bar{s}}$ gallbladder, together with barium studies of the $\vec{\nabla}$ small and large intestines, on three occasions over this period of time. They were all reported as $\infty$ normal. For the previous year attacks of pain $\vec{\circ}$ had increased in severity and frequency, becoming almost a daily occurrence.

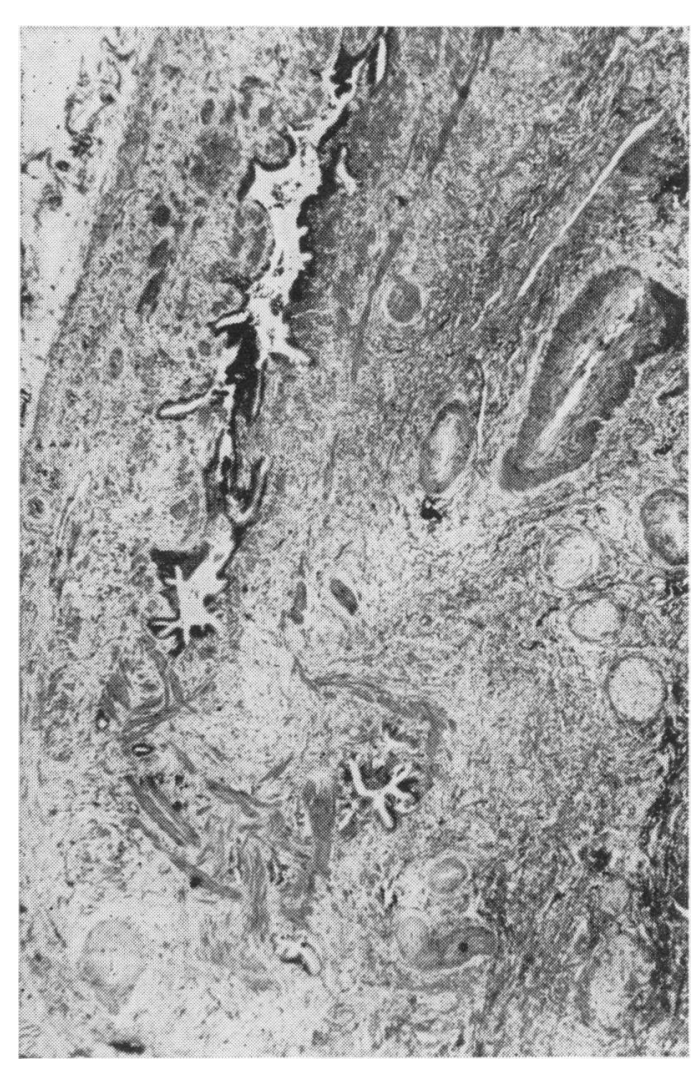

Fig. 3 Photomicrograph of a section of the gallbladder removed from patient 1 showing the proliferation of the mucosal and muscle layers with pseudoglandular formation (see also Figs. 4 and 5).

Physical examination was unremarkable apart $\underset{2}{2}$ from slight right upper quadrant tenderness. Liver function tests were all within normal limits. A barium study of the oesophagus, stomach, $\stackrel{+}{+}$ small and large intestines revealed only a small ${ }_{0}$ sliding hiatus hernia without demonstrable reflux. $\frac{\overrightarrow{0}}{\mathrm{D}}$ A cholecystogram (Fig. 1) showed normal con- $\frac{\rho}{1}$ centration of contrast material but the shape of $\stackrel{\varnothing}{\circ}$ the gallbladder was altered by a narrow area just $\overline{2}$ proximal to the fundus. At the fundus there was 8 an umbilicated filling defect suggestive of an adenomyoma. No calculi could be seen. Following a fatty meal there was normal contraction of the 
gallbladder but the defect at the fundus remained visible.

Review of previous cholecystograms performed 12, 11, and seven years earlier (Fig. 2) showed that a similar filling defect at the fundus of the gallbladder had been present for the whole of that time but on each occasion the gallbladder had concentrated the contrast material well and contraction after a fatty meal was normal.

A cholecystectomy was carried out. The serosal surface of the gallbladder was normal as were the extrahepatic ducts. The lesion at the fundus was found to be an adenomyoma (Fig. 3). Characteristic changes of adenomyomatosis consisting of hyperplastic muscle bundles, areas of proliferating mucosa forming gland and cyst-like structures, and a scattered chronic inflammatory cell infiltration extended around the macroscopic lesion for approximately $0.5 \mathrm{~cm}$. The rest of the gallbladder wall was normal and there were no calculi.

Following cholecystectomy, the patient lost all her symptoms. When seen a year later she remained asymptomatic.

\section{Case 2}

L.P., a man aged 43 years, complained of right upper quadrant pain for two years always in association with meals. The pain was sudden in onset, steady in intensity during its duration, but did not radiate. There had been no episodes of jaundice, fever, or change in colour of stools or urine. There was a past history of amoebic dysentery. Physical examination showed no abnormality, and liver function tests were all within normal limits. A cholecystogram showed

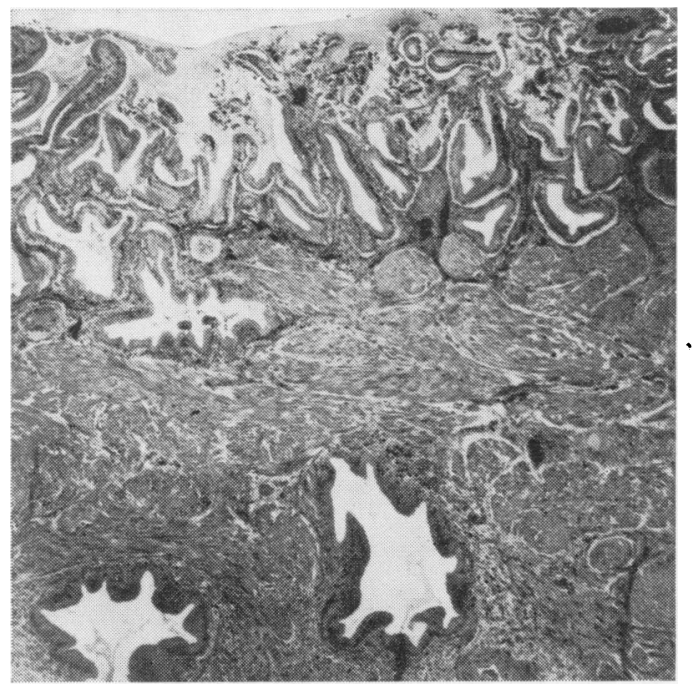

Fig. 4 Photomicrograph of a section of the gallbladder (patient 2). a dumbbell-shaped gallbladder with multiple Rokitansky-Aschoff sinuses filled with contrast material adjacent to the gallbladder lumen. No calculi were visualized. After a fatty meal the gallbladder contracted normally.

Cholecystectomy was carried out. On microscopy the wall of the gallbladder showed the typical changes of adenomyomatosis (Fig. 4). There were no calculi. Following cholecystectomy this patient lost all his symptoms. Fifteen months after the operation he reported that there had been no recurrence of his previous trouble.

\section{Case 3}

W.S., a man aged 54 years, complained of right upper quadrant pain after meals over a period of four years. The pain was poorly described but it did seem to be steady in intensity when present although not radiating. The attacks of pain were increasing in severity and frequency, each episode lasting about one hour. There was no history of jaundice, fever, dark urine, or pale stools. The patient was a diabetic. Physical examination showed no abnormalities. Liver function tests were all within normal limits. Cholecystogram showed a non-functioning gallbladder.

Cholecystectomy was carried out. The gallbladder contained a small, firm, brown, ovoid mass, $1.5 \mathrm{~cm}$ in diameter, in the ampulla extending into the cystic duct. The gallbladder wall immediately surrounding this mass was thickened to $4 \mathrm{~mm}$. The rest of the wall appeared normal. On microscopy (Fig. 5), the ampullary mass was composed of a fibromuscular stroma embedded in which were glandular structures lined by columnar cells. The mucosal surface showed prominent papillary

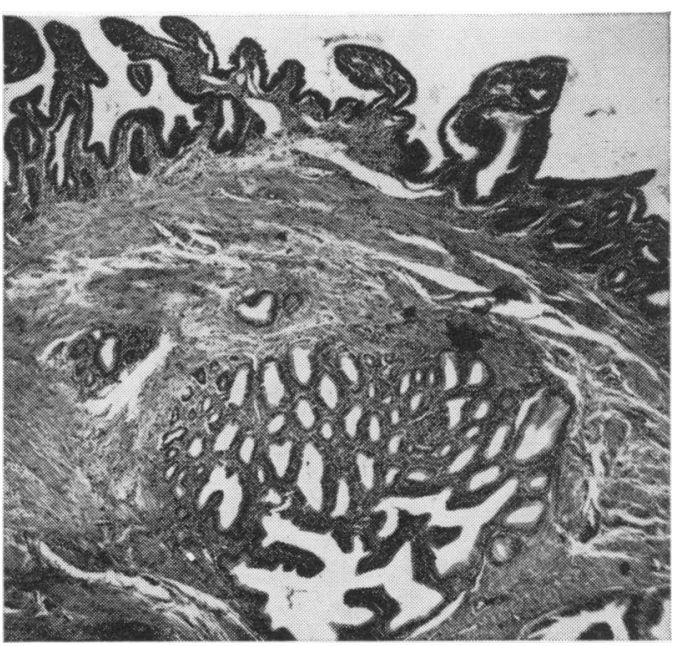

Fig. 5 Photomicrograph of a section of the gallbladder (patient 3). 
formations, and numerous Aschoff-Rokitansky sinuses were present in the surrounding gallbladder wall. The appearances were those of adenomyomatosis. After operation this patient was free of symptoms during a follow-up period of five years.

\section{Case 4}

B.W., a woman aged 69 years, had a 15 -year history of attacks of vague upper abdominal pain usually occurring after meals without jaundice, episodes of fever, dark urine, or pale stools. Cholecystograms on numerous occasions had shown a filling defect in the gallbladder fundus but there had been good concentration and the contraction of the gallbladder after fatty meals appeared normal. No calculi had been seen. A gastric resection had been performed for a duodenal ulcer 40 years before.

Physical examination showed no abnormality.

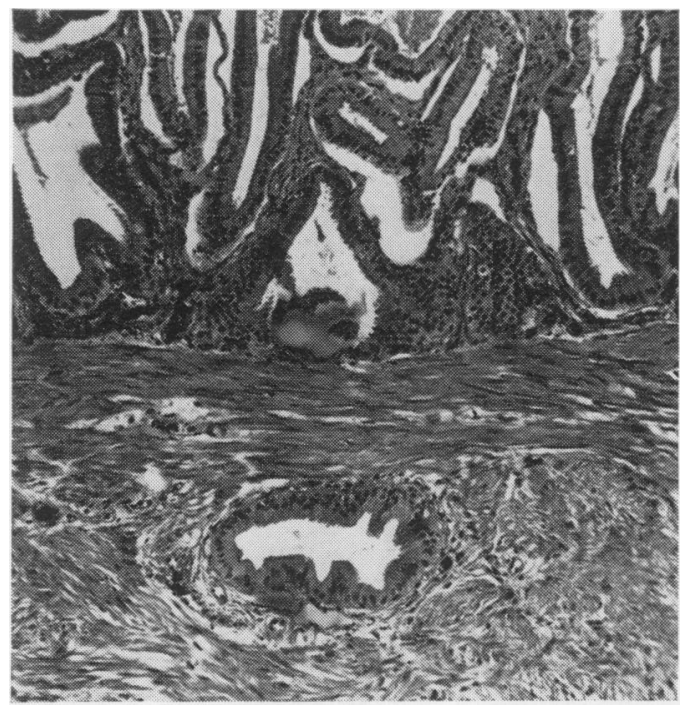

Fig. 6

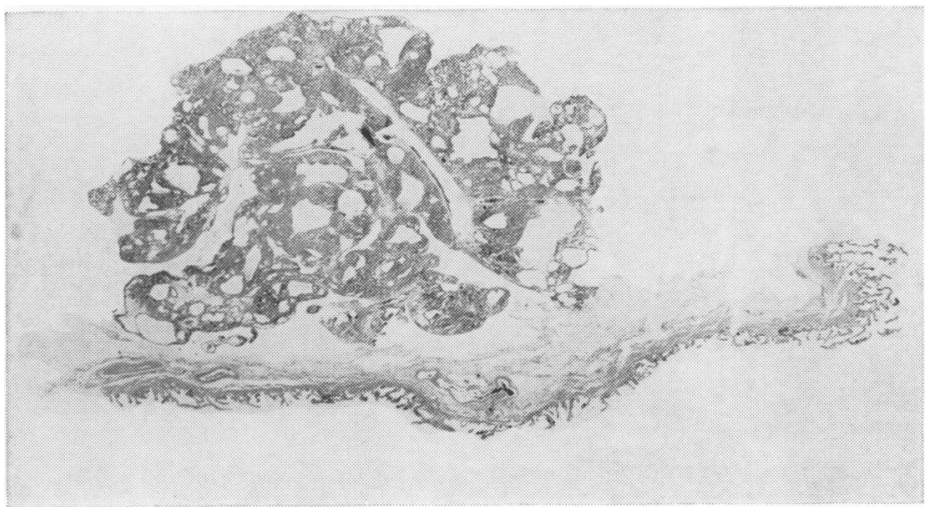

Fig. 7
Liver function tests were all within normal limits. Cholecystectomy was carried out. The gallbladder was found to contain a small button of tissue at $\stackrel{?}{\Rightarrow}$ the fundus of the gallbladder which on section $\stackrel{\vec{D}}{\vec{D}}$ proved to be an adenomyoma (Fig. 6). At the junction of the neck and the body there was a pedunculated polypoid mass $2.2 \mathrm{~cm}$ in diameter (Fig. 7), which on section (Fig. 8) consisted of an elaborate series of compact tubular and glandular \& structures. The cuboidal epithelium lining the glands was generally uniform with regular basal nuclei but in some areas there was crowding of cells with disruption of the basement membrane. Many glands were apposed 'back to back' without intervening stroma. Ball-like syncytial clusters of spindle-shaped cells with occasional mitoses could also be seen. Morphologically the process was that of a low-grade adenocarcinoma arising in a polypoid adenoma. Serial sections of the base of attachment showed no evidence of invasion.

Following the cholecystectomy, the patient became symptom-free and four years later showed no evidence of malignant disease.

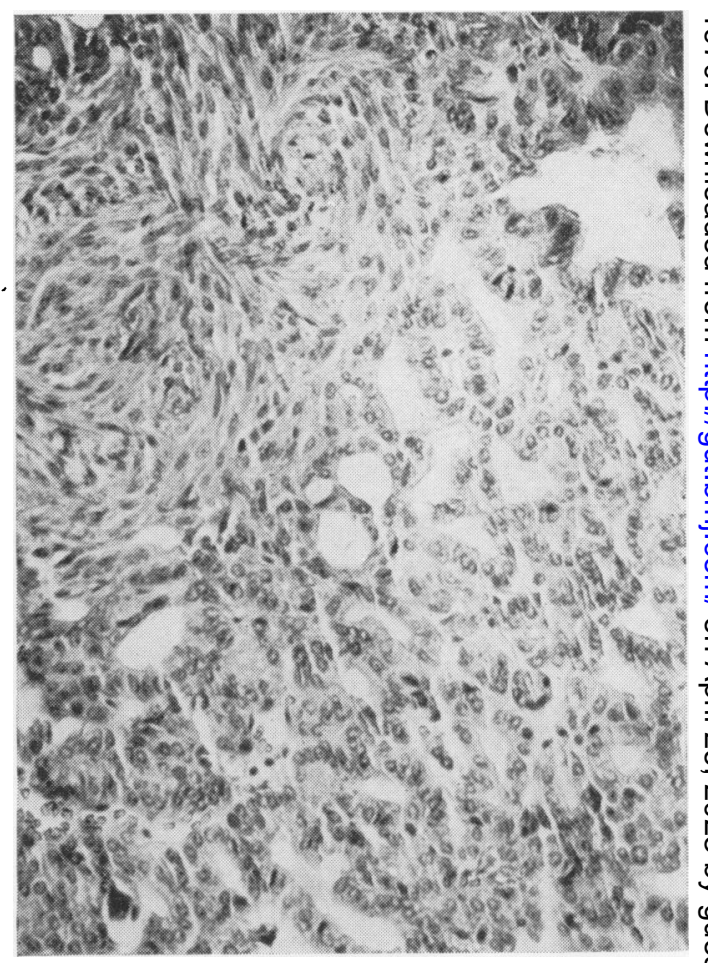

Fig. 8

Fig. 6 Photomicrograph of a section through the area of adenomyomatosis in patient 4.

Fig. 7 Low-power view of a section through the adenoma from patient 4.

Fig. 8 Photomicrograph of the adenoma shown in Fig. 7 demonstrating changes of adenocarcinoma. 
Case 5

B.R., a woman aged 47 years, had a 20 -year history of postprandial epigastric discomfort, particularly after drinking milk. Radiological investigation on several occasions had revealed a small hiatus hernia. A cholecystogram had shown a probable adenomyoma at the fundus of the gallbladder with multiple Rokitansky-Aschoff sinuses. A severe and sudden attack of epigastric pain radiating into the right upper quadrant and of constant intensity accompanied by nausea and vomiting precipitated admission to hospital.

Physical examination on admission revealed only right upper quadrant tenderness. There was no fever and liver function tests were all within normal limits.

A cholangiogram showed prompt visualization of the gallbladder and common bile duct. The contour of the fundus was irregular and, again, contrast-filled Rokitansky-Aschoff sinuses could be seen projecting beyond the lumen. Following a fatty meal the gallbladder contracted normally. No calculi were seen.

Cholecystectomy was carried out. The gross specimen showed a smooth serosal surface with no adhesions. No calculi were present. There was a small, button-like thickening, $0.5 \mathrm{~cm}$ in diameter, at the fundus of the gallbladder, which on section proved to be a typical adenomyoma.

After cholecystectomy this patient lost all her symptoms. She was followed up for four years.

\section{Case 6}

J.K., a woman aged 46, had experienced several attacks of epigastric and substernal pain over a two-year period, occurring usually after meals. The pain was constant when present, lasting two to three hours at a time, but not radiating. There had been no episodes of jaundice, fever, or change in colour of stools or urine.

Physical examination revealed tenderness in the right upper quadrant only. There was no fever and liver function tests were all within normal limits. Oral cholecystography demonstrated a marginal irregularity consisting of RokitanskyAschoff sinuses without evidence of calculi. Following a fatty meal there was complete emptying of the gallbladder.

Cholecystectomy was carried out. Microscopic section of the wall of the gallbladder showed the characteristic appearances of adenomyomatosis with a localized condensation at the fundus.

Following cholecystectomy, the patient lost all her symptoms. She was followed up for seven years.

\section{Comments}

The response to cholecystectomy shown by these patients suggests that the adenomyomatosis was the cause of symptoms in each case. The absence of cholelithiasis and of inflammatory changes make them unlikely sources of pain although it is possible that these factors were operative at an earlier stage in the disease process.

It is noteworthy that symptoms had been present in this group of patients for 11, 2, 4, 15, 20, and 2 years respectively. Each patient had undergone radiological investigation of the whole gastrointestinal tract on more than one occasion in addition to several cholecystograms. In all instances the absence of gallstones and the ability of the gallbladder to concentrate and excrete dye adequately had delayed surgical treatment. It is not uncommon for such patients to undergo repeated investigation in an attempt to find an alternative explanation for the pain which may finally come to be regarded as functional in origin.

On the basis of observations on the courses of the patients in the current report, it is recommended that when symptoms suggestive of biliary tract disease occur in association with the cholecystographic appearances of acalculous adenomyomatosis, cholecystectomy should be advised if there is no other obvious cause for the symptoms. When the condition is discovered incidentally, in the absence of definite symptoms, the problem becomes similar to that of the silent gallstone. Whether or not surgical treatment is advised will then depend upon such factors as the age of the patient, the coexistence of other diseases, and the ready accessibility of medical care should complications arise.

The mechanism by which pain is produced is not entirely clear. It is presumably related to gallbladder contraction since patients often comment that their symptoms were reproduced during cholecystography following ingestion of the fatty meal. Jutras and Lévesque (1966) have made the observation that films taken when pain is experienced show overdistension of the cystic and common bile ducts. They believe that pain is produced by excessive neuromuscular activity of the hyperplastic gallbladder wall resulting in vigorous contraction and distension of the ducts. In support of this contention it is commonly observed that, following a fatty meal, contraction of the gallbladder takes place earlier than usual, frequently within five minutes, and also that it appears to contract to a smaller size than usual.

It has been suggested that excessive motility may be related to a proliferation of subepithelial neural elements accompanying the mucosal and muscular hyperplasia. This type of superficial neuromatosis was first described in conjunction with cholesterolosis of the gallbladder (Riopelle, 1942) but it has also been demonstrated in adenomyomatosis (Jutras et al, 1960). Others have denied the importance of this appearance which they attribute to a condensation rather than a proliferation of nerve fibres (Lubera, Climie, and Kling, 1967). 
The presence of a carcinoma in patient 4 again raises the possibility that adenomyomatosis may predispose to malignant change. Previous discussions of this topic have been obscured by misinterpretation of the histological features of adenomyomatosis and by differences in terminology. Sutherland (1898) first used the term adenomyoma for the localized form of the condition. Since that time other writers have referred to the same lesion as an adenoma or an adenofibroma or an epitheliomyoma. All these terms are misleading because they imply, quite falsely, that the tissue changes are neoplastic in origin. The Rokitansky-Aschoff sinuses are not true glands so that the appellation cholecystitis glandularis proliferans is equally unsuitable.

Malignant degeneration within an area of adenomyomatosis has not been described. Because of the extension of the RokitanskyAschoff sinuses into the muscle layer the lesion may resemble invasion by adenocarcinoma. The microscopic picture may also be confusing because of the different planes in which the cells may be cut. These factors almost certainly account for the occasional impression of malignant change, as in the case reported by Eiserth (1938). True adenomatous polyps, like the one removed from patient 4 in the present series, are rare. This lesion has been considered to be precancerous by some authors (Wellbrock, 1934; Kane, Brown, and Hoerr, 1952; Tabah and McNeer, 1953; Ochsner and Carrera, 1956) whilst others have maintained that such polyps are always benign (Phillips, 1933; Shepard, Walters, and Dockerty, 1942; Eelkema, Hodgson, and Stauffer, 1962). This difference in opinion reflects the general difficulty in defining malignant change in adenomatous polyps arising from all mucosal surfaces. The adenoma in the patient in the present series showed many features, described in the case report, which warranted a diagnosis of adenocarcinoma.
It is likely that the tumour was a coincidental finding. Nevertheless, carcinoma usually arises? in an abnormally functioning gallbladder, most commonly in association with gallstones. It $\stackrel{\overrightarrow{0}}{\overrightarrow{3}}$ remains possible, therefore, that the disturbanceo in physiological function resulting from the adeno-음 myomatosis may also have contributed to an $\frac{\rho}{5}$ alteration in cell growth within the adenoma.

References

Bricker, D. L., and Halpert, B. (1963). Adenomyoma of the gallbladder. Surgery, 53, 615-620.

Eelkema, H. H., Hodgson, J. R., and Stauffer, M. H. (1962). $\cdot$ Fifteen-year follow-up of polypoid lesions of the gall $\overrightarrow{\mathrm{N}}$ bladder diagnosed by cholecystography. Gastroenterology, $\overrightarrow{\vec{O}}$ 42,144-147.

Eiserth, P. (1938). Adenomyome der Gallenblase. Virchows Arch. ్ํ path. Anat., 302, 717-723.

Jutras, J. A., and Lévesque, H. P. (1966). Adenomyoma and adeno- 음 myomatosis of the gallbladder. Radiol. Clin. N. Amer., 4, 483-500.

Jutras, J. A., Longtin, M., and Lévesque, H. P. (1960). Hyperplastic cholecystoses. Amer. J. Roentgenol., 83, 795-827.

Kane, C. F., Brown, C. H., and Hoerr, S. O. (1952). Papilloma of

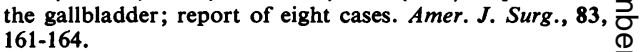

King, E. S. J., and MacCallum, P. (1931). Cholecystitis glandularis proliferans (cystica). Brit. J. Surg., 19, 310-323.

Le Quesne, L. P., and Ranger, I. (1957). Cholecystitis glandularis proliferans. Brit. J. Surg., 44, 447-458.

Lubera, R. J., Climie, A. R. W., and Kling, G. E. (1967). Cholecystitis and the hyperplastic cholecystoses: A clinical, radiologic and pathology study. Amer. J. dig. Dis., 12, 696-704.

Ochsner, S., and Carrera, G. M. (1956). Benign tumors of the gall bladder. Gastroenterology, 31, 266-273.

Phillips, J. R. (1933). Papilloma of the gallbladder. Amer. J. Surg., 21, 38-42.

Riopelle, J. L. (1942). Sur les proliférations nerveuses de la vésicule $\overrightarrow{\overrightarrow{0}}$ biliaire (neuromatoses vésiculaires). J. Hôtel-Dieu Montréal, 11, 3-76.

Sutherland, L. R. (1898). Small adeno-myoma of the gall-bladder. Glasg. med. J., 50, 216-217.

Shepard, V. D., Walters, W., and Dockerty, M. B. (1942). Benign neoplasms of the gallbladder. Arch. Surg., 45, 1-18.

Tabah, E. J., and McNeer, G. (1953). Papilloma of the gallbladder with in situ carcinoma. Surgery, 34, 57-71.

Wellbrock, W. L. A. (1934). The occurrence and possible significance of adenoma of the gallbladder. Amer. J. Surg., 23, 358-360. 